\title{
Silicon-Supported Membranes for Improved Large-Area and High-Density Micro/Nanostencil Lithography
}

\author{
Marc A. F. van den Boogaart, Lianne M. Doeswijk, and Jürgen Brugger
}

\begin{abstract}
In this paper, the fabrication and use of stencils for full-wafer scale shadow mask (stencil) lithography is described. The stencils fabricated via microelectromechanical systems are mechanically stabilized and show clearly reduced stress-induced membrane deformation, which translates into a more accurate surface pattern definition. Solid-state SiN membranes $500 \mathrm{~nm}$ thick and up to $1 \mathrm{~mm}^{2}$ in size having a 20 - $\mu \mathrm{m}$-thick silicon support rim following the outline of the stencil apertures were fabricated in a 100-mm $\mathrm{Si}$ wafer. The minimum aperture size presented in this paper is $3 \mu \mathrm{m}$. The increase of membrane stability was confirmed by depositing a highly stressed 35-nm-thick chrome layer. The results demonstrate a stability increase of the Si-supported compared to nonsupported membrane with identical shape by up to $89 \%$ as measured by the reduced out-of-plane deflection of overhanging membrane sections. Comparison by scanning electron microscopy and atomic force microscopy of the resulting micropatterns obtained by $\mathrm{Cr}$ deposition through both unsupported and Si-rim supported stencils shows better edge sharpness and clearer spatial details for surface patterns deposited through the stabilized stencil compared to those deposited through the nonsupported stencil. The improved stabilized stencils allow for large-area high-density surface patterning while maintaining membrane stability and pattern definition during stencil lithography.

[2006-0003]
\end{abstract}

Index Terms-Local deposition, mechanical stabilization, nanostencil, shadow mask, stencil lithography, stress-induced deformation.

\section{INTRODUCTION}

A $\mathrm{N}$ increased demand for micro- and nanometer scale patterning on "unconventional" surfaces and/or non-integrated-circuit applications has given rise to new and alternative patterning technologies. These new methods include indentation of polymers by nanoimprint lithography (NIL) [1], [2], local deposition of molecules via a stamp by micro contact printing $(\mu \mathrm{CP})$ or soft-lithography [3], [4], or via dip-pen nanolithography [5], nanoscale fluidic dispensing [6] and localized material deposition through ultraminiature shadow masks (nanostencils) [7]-[15]. Of these above-mentioned alternative

Manuscript received February 1, 2006; revised July 13, 2006. This work was supported in part by OFES within the European Commission Project NaPa under Contract NMP4-CT-2003-500120 and by the Swiss National Science Foundation Project NANO-IC under Contract 200021-101847. Subject Editor H. Fujita.

The authors are with Ecole Polytechnique Fédérale de Lausanne, Laboratoire de Microsystèmes, CH-1015 Lausanne, Switzerland (e-mail: marc.vandenboogaart@epfl.ch; liannedoeswijk@hotmail.com; juergen. brugger@epfl.ch).

Digital Object Identifier 10.1109/JMEMS.2006.885981 patterning techniques, NIL, $\mu \mathrm{CP}$, and stencil lithography are replication methods (as opposed to serial patterning methods) with the potential of full-wafer high-throughput parallel pattern formation. In addition, stencil lithography has the advantage of being a direct vacuum patterning technology in a single step process, i.e., a controlled amount of the desired material is directly deposited only where necessary through the stencil apertures without the need for further processing steps as seen, for example, in photolithography and NIL. An additional advantage of stencil lithography over other patterning methods is its possible noncontact application avoiding cross-contamination or surface damage in case of fragile surfaces, whereas NIL needs hard contact and $\mu \mathrm{CP}$ needs soft contact between master and substrate to transfer the pattern successfully. These specifics make stencil lithography applicable to full-wafer nonplanar surfaces such as MEMS devices and complementary metal-oxide-semiconductor chips [16] that can also be either mechanically unstable, such as cantilevers and membranes, and/or functionalized surfaces, such as, e.g., self-assembled monolayers [12].

Stencil lithography makes use of reusable, miniaturized shadow masks using thin solid-state membranes (e.g., SiN thinner than $500 \mathrm{~nm}$ for submicrometer apertures) to accommodate nanoaperture fabrication. Recently, we have introduced a process for high-throughput fabrication of stencil membranes $\left(1 \times 1 \mathrm{~mm}^{2}\right)$ on a $100 \mathrm{~mm}$ wafer using a combination of deep ultraviolet (DUV) lithography and standard microelectromechanical system (MEMS) processes [17]. DUV lithography enables full wafer exposure for high-throughput micro/nanoaperture definition. This type of full wafer stencil allows for direct, resistless patterning of mesoscopic surface structures (200 nm up to several hundred micrometers) on a full-wafer scale by any material flux on a large variety of substrates. Fig. 1 shows such a stencil on a $100 \mathrm{~mm}$ wafer and the resulting surface patterns on a $\mathrm{Si}$ wafer after $100 \mathrm{~nm} \mathrm{Al}$ deposition. The deposition through stencils suffers, however, from drawbacks such as clogging of apertures and membrane deformation due to the deposition-induced stresses. Clogging occurs as the evaporated material is also deposited inside the apertures. This phenomenon changes the geometrical shape of the aperture during the deposition process and leads to deformation of the deposited pattern and eventually to the complete closure of the aperture. Stencil cleaning in between deposition process and/or reducing material adhesion to the membrane [18] will be necessary to maintain accurate pattern replication 


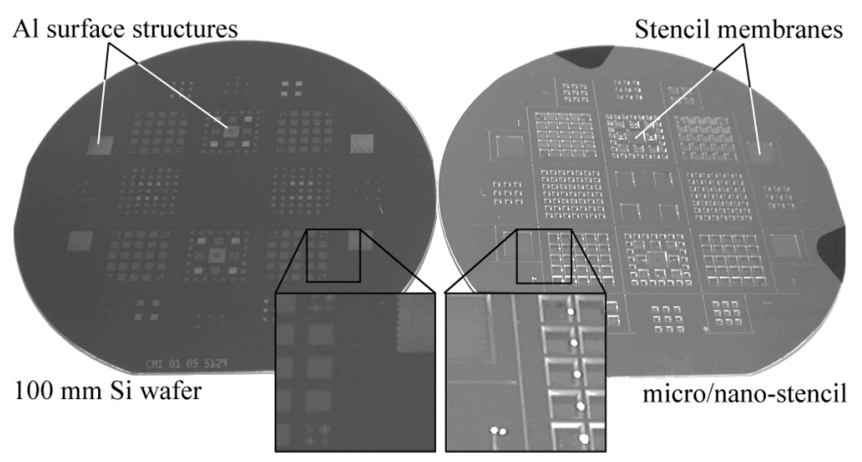

Fig. 1. Optical picture of a $100-\mathrm{mm}$ micro/nanostencil containing membrane sizes ranging from $1 \times 1 \mathrm{~mm}^{2}$ to $4 \mathrm{~mm} \times 4 \mathrm{~mm}^{2}$ and a Si substrate after evaporation of 100-nm-thick Al.

and stencil reusability. In this paper, we focus on the issue of membrane deformation resulting from thin-film related stresses within the material deposited on the stencil membrane and its effect on surface structure definition. The internal stress in the deposited material is caused by thermal stress and deposition related intrinsic stresses [19]. The consequent changes in membrane aperture geometry and in the gap between the stencil membrane and substrate result in pattern distortion and blurring (i.e., reduced edge sharpness and limited spatial resolution) of the deposited surface structures. Yotsuya and Makigaki [20] proposed an interesting solution where a stencil is kept in contact with a substrate using a magnetic layer that is deposited onto the stencil. The stencil is attracted towards the substrate by a magnet, situated on the other side of the substrate. For very thin membranes, the magnetic layer might cause additional bending of thin stencil membranes, hence we propose here an alternative solution where the initial gap is maintained and where stencil deformation is reduced via pure, passive, local mechanical reinforcements.

Fig. 2 shows the effect of membrane deformability on the surface structures in three situations. The first image [see Fig. 2(a)] shows an ideal situation of a stencil deposition process in which the stencil membrane does not deform. This is the situation in the case of the deposition of a thin layer of low-stress material, or if the membrane contains equally sized and spaced apertures i.e., sieves, making the stencil intrinsically stable. In this situation, the dimensions and shape of the deposited surface patterns can be described by a simple model based on geometrical considerations [21]. The second image [see Fig. 2(b)] shows the deposition process using a nonsupported stencil in which the membrane aperture is deformed and the gap is increased during deposition, for instance, due to high-stress material deposition or unstable membrane aperture configurations. Fig. 2(c) shows the improved stabilized stencil deposition process in which the deformation of the thin membrane due to thin-film related stresses is limited by the stabilization structures. In addition, these stabilization structures will allow for stencil cleaning in between deposition processes.

We need to consider several conditions when developing stabilization structures into stencil membranes for exact pattern replication. First, the stabilization structures need to be located on the backside (i.e., the side facing the incoming material flux) of the stencil membrane, assuring a close contact between sub-

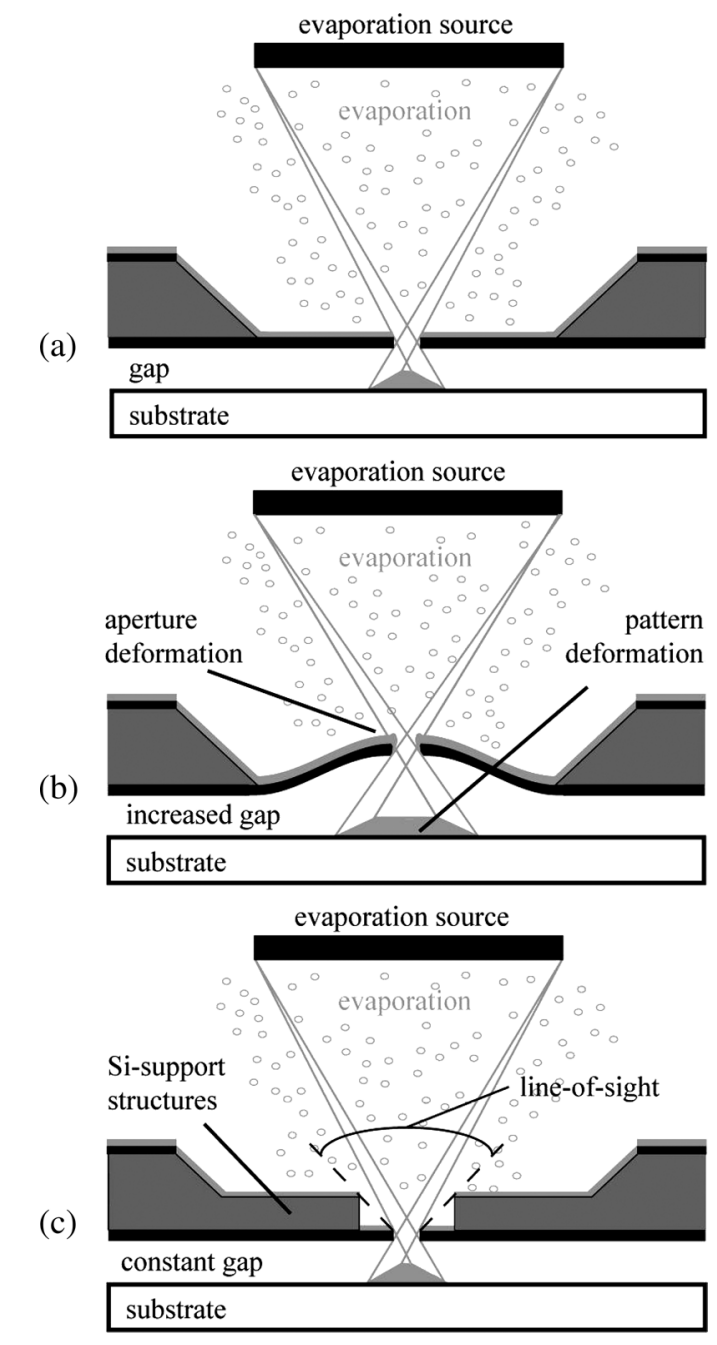

Fig. 2. Schematic representation of a stencil membrane in close proximity of a substrate during evaporation. (a) Ideal stencil deposition (nondeformed membrane). The membrane stays unchanged and does not add to pattern deformation. (b) Stencil deposition using a nonsupported stencil membrane (deformed membrane). The deformed membrane has both an increased gap and aperture deformation, resulting in uncontrollable pattern deformation. (c) Si-supported stencil deposition. The membrane is locally supported while not affecting the line-of-sight of deposition. The membrane deformation and pattern blurring are reduced.

strate surface and stencil membrane in order to minimize the gap. Second, the stabilization structures should not interfere with the material transport from the source through the apertures, i.e., the line-of-sight of the apertures to the material source should not be obstructed [see Fig. 2(c)]. Third, the stabilization structures should be easily adaptable to the arbitrary geometry of membrane apertures. To date, efforts have been reported to stabilize membranes locally by introducing stabilization structures. For instance, Kim et al. [7] used focused ion beam (FIB) milling to locally thin down a 500-nm-thick membrane to below $100 \mathrm{~nm}$, enabling the creation of $<100 \mathrm{~nm}$ sized apertures while the rest of the 500-nm-thick membrane remains stable. This method enables local stabilization of nanosized apertures, but the sequential nature of FIB-milling reduces the throughput. Furthermore, the stability of this membrane is determined by the original membrane thickness. Kuiper et al. [22], [23] defined membrane stabilization structures for microfiltration applications by a wet etching process $(\mathrm{KOH})$ that depends on the 


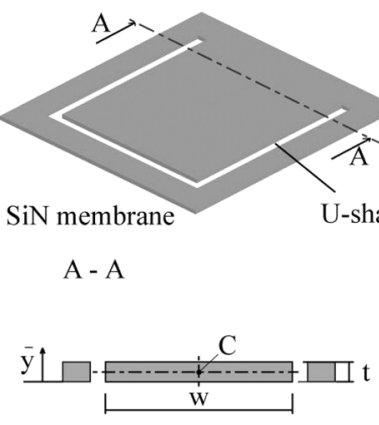

(a)
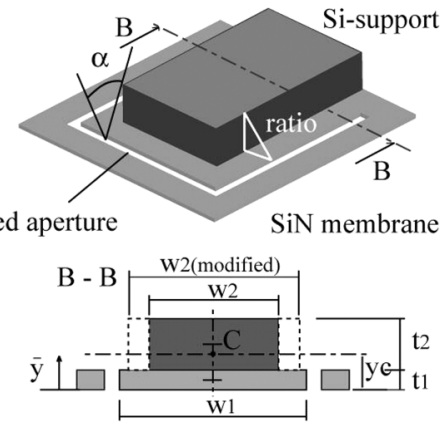

(b)

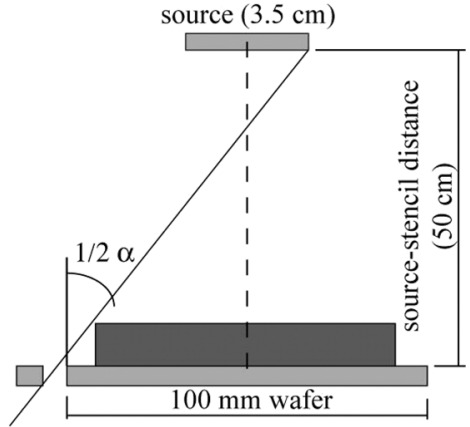

(c)

Fig. 3. Schematic representation of a membrane containing a U-shaped aperture and its cross-section. (a) A 500-nm-thick unsupported SiN membrane. (b) A 500-nm-thick SiN membrane supported by $20-\mu \mathrm{m}$-thick Si. The width of the cantilever is $50 \mu \mathrm{m}$. The Si-support width (30 $\mu \mathrm{m}$ ) is modified according to the ratio of the Young's modulus of Si and SiN [see (2)]. The line-of-sight angle $\left(>16^{\circ}\right)$ and ratio (maximum $\left.7: 1\right)$ are shown in Fig. 3(b).

crystal orientation of the substrate $(\mathrm{Si})$. Since these structures follow the crystal orientation, they can only be applied to limited stencil geometries. Hammer et al. [24] used support ribs fabricated by milling. The support rib structures interfered with the deposition line-of-sight and thus resulted in reduced pattern definition and uniformity. Both mentioned methods therefore do not fulfill the ideal conditions for stencil lithography, e.g., aperture adaptable stabilization structures and line-of-sight. Therefore, we have developed new stabilization schemes based on MEMS processes that fulfill all above-mentioned conditions. We have recently presented an alternative scheme for thin $\mathrm{SiN}$ membrane stabilization based on the introduction of corrugations within the membrane [11]. These corrugated SiN membranes are fabricated by adding one process step to the standard stencil fabrication process. This simple process resulted in considerably more stable membranes, yielding well-defined surface patterns. However, the ratio (height : width) of the corrugations cannot be varied unlimitedly due to the thin corrugated wall thickness (determined by the membrane thickness). This limits the total increase of membrane stiffness that can be achieved with corrugations [11]. Therefore, we developed a second stabilization method with the possibility for the fabrication of even more stable membranes, opening the possibility for larger area stencil membranes and stencil cleaning.

In this paper, a new fabrication process is presented which allows the integration of geometrically adaptable Si-support structures onto thin SiN membranes while maintaining line-of-sight and possible close contact of the stencil with the substrate and thus fulfilling all the previously stated conditions. These stabilized stencils can be used in stencil lithography but also in, e.g., ion projection lithography [14] and microfiltration [23]. To verify the improved stencil stability, we have deposited a highly stressed 35-nm-thick chrome layer (as a worst case scenario) onto and through both (a) an improved stabilized stencil (Si-supported) and (b) a nonstabilized stencil (standard) onto a silicon substrate. Both the membrane deflection and the corresponding deposited surface structures were analyzed.

\section{Membrane Design AND DeFlection Analysis}

In order to be able to create arbitrary surface structures by stencil lithography, the membrane needs to contain the corresponding arbitrary apertures. In some specific cases these aper- tures might have the shape of long slits, slits with corners, or U-shape openings. Such a membrane structure mechanically resembles a cantilever as shown in Fig. 3. The deflection of this cantilever during thin-film deposition increases the gap between the substrate and membrane, resulting in surface pattern deformation and blurring, i.e., reduced sharpness of edges and limited spatial detail. To develop an improved stabilization scheme, we looked at the deflection of a thin cantilever under the influence of surface stress. The free-end displacement of a cantilever under the influence of a thin, stressed layer can be approximated using the Stoney formula [25]. In (1), we have substituted the moment of inertia $I_{c}$, which is the structural property directly related to stiffness or deformability, for a rectangular cross-section of a cantilever in the Stoney formula

$$
\delta=\frac{\sigma_{f}\left(1-v_{c}\right) w t_{f} t_{c} l^{2}}{4 E_{c} I_{c}} .
$$

Equation (1) gives the free-end displacement of a cantilever as a function of thin-film stress and the moment of inertia, in which $\delta$ is the free-end displacement, $\sigma_{f}$ is the thin-film stress, $v_{c}$ is the Poisson's ratio of the cantilever material, $w$ is the width, $t$ is the thickness, $l$ is the length, $E$ is the Young's modulus, and $I$ is the moment of inertia. The subscript $c$ is for the cantilever and the subscript $f$ is for the film.

According to (1), to reduce the deflection of an arbitrary cantilever independent from the thin-film deposition process, we could increase the moment of inertia. Because the membrane apertures are defined by the need for specific surface structures $\left(w, l\right.$, and $\left.t_{f}\right)$, only the thickness of the cantilever can be increased freely to increase $I$, as long as the condition for the line-of-sight is respected Fig. 2(c). In the deposition equipment we used for these experiments, with the given geometry, a minimum open angle $\alpha$ is required for a full line-of-sight deposition [see Fig. 3(b) and (c)], i.e., in order to avoid obstruction of the material flux by the stabilization rims. This angle $\alpha$ corresponds to a ratio between the membrane thickness and the aperture size. We have shown that $250 \mathrm{~nm}$ apertures can be fabricated in a 500-nm-thick SiN membrane [17], which corresponds to an aspect ratio of $2: 1$. Obtaining an aspect ratio of more than $2: 1$ in nanoaperture definition is difficult to realize by standard MEMS processing. As already observed by Graff et al. [13], the 
aspect ratio in the stencil has a significant effect on the profile of the material being deposited. Furthermore, this aspect ratio has a direct influence on the pattern size uniformity across an, e.g., $100 \mathrm{~mm}$ stencil. For example, based on geometrical calculations, the structure size deposited through a $1 \mu \mathrm{m}$ aperture placed in the center compared to one deposited on the edge of a $100 \mathrm{~mm}$ stencil differs in size due to a partial shadowing of the membrane. If we assume the stencil in contact with the substrate at $50 \mathrm{~cm}$ from the source $3.5 \mathrm{~cm}$ in size, then a $5-\mu \mathrm{m}$-thick membrane with a $1-\mu \mathrm{m}$-wide aperture (ratio $5: 1$ ) results in a pattern size of approximately $675 \mathrm{~nm}$ at the wafer edge. Correspondingly, a $1 \mu \mathrm{m}$ aperture in a 500-nm-thick membrane results in a pattern size of approximately $900 \mathrm{~nm}$ at the wafer edge. Working with full wafer sized stencils $(100 \mathrm{~mm})$ with apertures ranging from $250 \mathrm{~nm}$ up to several hundred micrometers requires a stabilization scheme that respects this aspect ratio.

In this paper, we add a Si-support structure on top of the SiN membrane in order to increase its moment of inertia. This $\mathrm{Si}$ support is following the aperture edge of the $\mathrm{SiN}$ membrane at a fixed distance, defining an effective aspect ratio of $2: 1$ for the line-of-sight criteria. Concretely, in this contribution, we realized a $20-\mu \mathrm{m}$-thick Si support with a distance of $10 \mu \mathrm{m}$ to the aperture edge. This stabilization can be applied to the entire membrane for any kind of shape while maintaining the line-of-sight unobstructed. The concept is also scalable to smaller dimensions.

The moment of inertia for, e.g., a 500-nm-thick and 50- $\mu \mathrm{m}$ -wide cantilever is $0.52 \mu \mathrm{m}^{4}$ [see Fig. 3(a) and (2)]. When we add a $20-\mu \mathrm{m}$-thick and $30-\mu \mathrm{m}$-wide Si-support structure onto the 50- $\mu \mathrm{m}$-wide SiN cantilever (respecting the $10 \mu \mathrm{m}$ distance from the edge), $I$ increases to $3.03 \cdot 104 \mu \mathrm{m}^{4}$, an increase of almost a factor of $10^{5}$. A Young's modulus ratio of $180 / 250$ $\left(E_{\mathrm{Si}} / E_{\mathrm{SiN}}\right)$ was used for calculating an equivalent cross-section in which the width of the $\mathrm{Si}$-support parallel to the principal axis of bending is increased in the same ratio that $E_{\mathrm{Si}}$ has with $E_{\mathrm{SiN}}$ [see Fig. 3(b) and (2)] [26]

$$
I_{\text {totaal }}=\sum_{i=1}^{n} \frac{1}{12} \cdot w_{i} \cdot t_{i}^{3}+A_{i} \cdot d_{i}^{2} \text { and } y_{c}=\frac{\sum_{i=1}^{n} \vec{y}_{i} \cdot A_{i}}{\sum_{i=1}^{n} A_{i}} \text {. }
$$

Equation (2) is the moment of inertia for combined rectangular cross-sections in which $w$ is the width $[\mu \mathrm{m}], t$ the thickness $[\mu \mathrm{m}], A$ the area $\left[\mu \mathrm{m}^{2}\right], y$ the centroid position of each component, $y_{c}$ the position of the neutral axis $[\mu \mathrm{m}]$, and $d$ the distance of the centroid of the cross-section to the neutral axis or bending line $\left(y_{c}\right)[\mu \mathrm{m}]$.

\section{Fabrication of Silicon Supported SiN MEMbranes}

The silicon-supported microstencils were fabricated using standard MEMS processing. Fig. 4 shows a schematic overview of the fabrication process for a stabilized stencil. For simplicity reasons, the schematic fabrication overview of Fig. 4 does not illustrate lithographic process steps.

The process begins on a $100 \mathrm{~mm}$, double side polished, $380-\mu$ m-thick silicon wafer as illustrated in Fig. 4(a). Patterns for the membrane stabilization apertures were defined in two subsequent steps as shown in Fig. 4(b). First, the patterns were
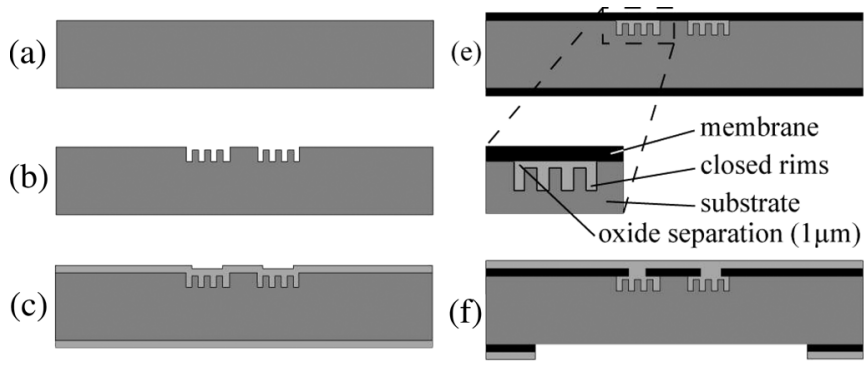

(d)

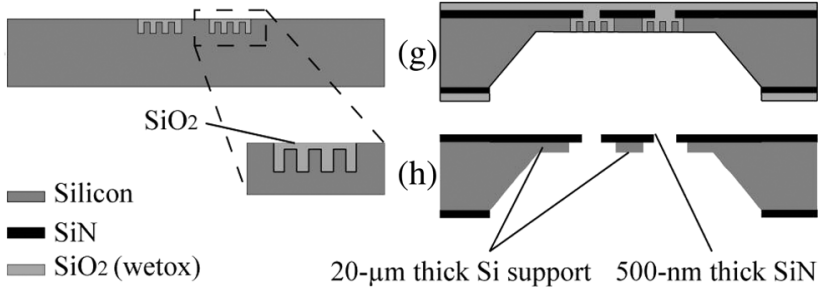

Fig. 4. Simplified schematic illustration of the fabrication process for a Si-supported stencil. (a) Process starts with a $380-\mu \mathrm{m}$-thick double side polished Si wafer; (b) support aperture definition by lithography and dry etch transfer; (c) support aperture sacrificial oxidation; (d) chemical mechanical polishing until bulk Si surface; (e) 500-nm-thick LPCVD low-stress SiN deposition; (f) dry etch transfer of membrane patterns and backside windows into $\mathrm{SiN}$ followed by LTO deposition (removed from the back side); (g) $\mathrm{KOH}$ etching of the bulk Si until a $20-\mu \mathrm{m}$-thick Si layer is left; and (h) final membrane release using $\mathrm{BHF}$.

defined in a 1.7- $\mu \mathrm{m}$-thick positive photoresist (S1818, Shipley, Conventry, U.K.) by means of conventional photolithography (MA-150 aligner, SUSS MicroTec, Garching, Germany). The $\mathrm{Si}$ was etched using inductively coupled plasma (ICP) anisotropic etching for a depth of $1 \mu \mathrm{m}$. This preetch allows for complete separation of the $\mathrm{SiN}$ membrane from the sacrificial $\mathrm{Si} / \mathrm{SiO}_{2}$ as will be described later. Then, a second mask pattern was used to etch 20- $\mu \mathrm{m}$-deep trenches (Alcatel 601E: Bosch process, $\mathrm{SF}_{6} 300 \mathrm{sccm}$ pulsed, $\mathrm{C}_{4} \mathrm{~F}_{8} 150 \mathrm{sccm}$ pulsed, $20^{\circ} \mathrm{C}$, $1500 \mathrm{~W}$ ). This pattern consists of a $4-\mu \mathrm{m}$-wide continuous trench following the outer edge of the support aperture and a grid of $4-\mu \mathrm{m}$-wide trenches with a spacing of $15 \mu \mathrm{m}$. A scanning electron microscopy (SEM) image of such a pattern is shown in Fig. 4. Oxidation was performed in a pyrogenic oxidation furnace $\left(\mathrm{H}_{2}=16 \mathrm{slm}, \mathrm{O}_{2}=8.3 \mathrm{slm}, 1250{ }^{\circ} \mathrm{C}\right.$, oxidation time $=542 \mathrm{~min}$ ) in order to refill the trenches with a sacrificial layer. The oxidation step fills the trenches and forms a uniformly closed surface layer [see Fig. 4(c) and Fig. 5 inset].

In Fig. 4(d), the bottom side $\mathrm{SiO}_{2}$ layer is removed using buffered hydrofluoric (BHF) acid $\left(\mathrm{NH}_{4} \mathrm{~F}(40 \%)\right.$ : $\mathrm{HF}(49 \%)$, ratio $7: 1)$ while protecting the front with resist $(3.7 \mu \mathrm{m}$ thick, S1818, Shipley). Then, the front side oxide is planarized using chemical mechanical polishing (CMP) (Steag Mecapol E 460, slurry: $30 \mathrm{~N} 50$, removal rate: $1700 \AA / \mathrm{min})$. The surface is polished until the surface of the wafer silicon surface is free from oxide except the oxide remaining in the stabilization apertures as defined beforehand. A 500-nm-thick low-stressed silicon nitride (LS-SiN) layer was then deposited with low-pressure chemical vapor deposition (LPCVD) (105 $\mathrm{sccm} \mathrm{SiH}_{2} \mathrm{Cl}_{2}$, $27 \mathrm{sccm} \mathrm{NH}$, $135 \mathrm{mTorr}, 838.5^{\circ} \mathrm{C}$, stress $200 \mathrm{MPa}$ tensile) as membrane material [see Fig. 4(e)]. The membrane apertures and the backside patterns were defined in a $1.7-\mu \mathrm{m}$-thick 


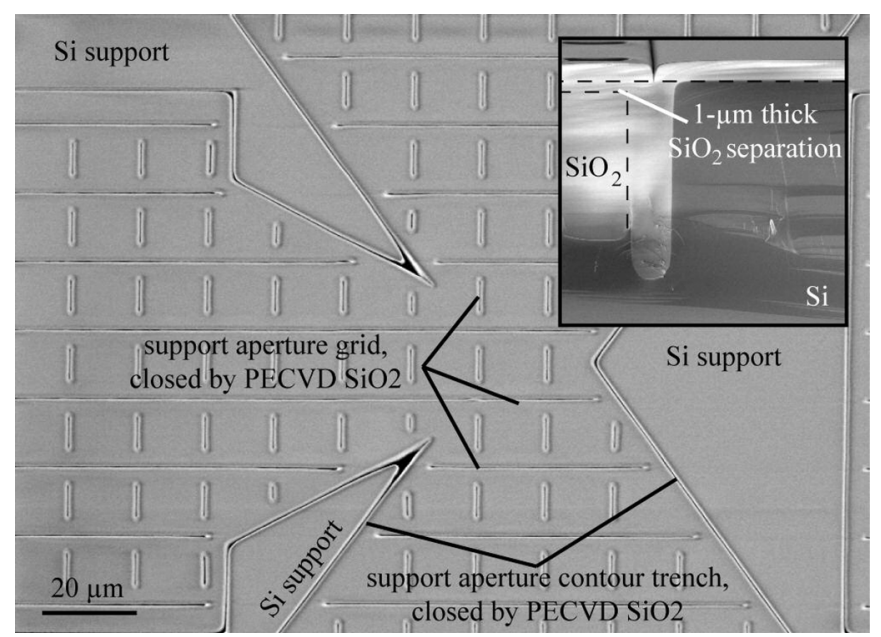

Fig. 5. A SEM of the wafer surface after wet oxidation of the support apertures. Image shows Si-support structures and oxidized trenches matching the stabilization apertures. Inlet shows a cross section after wet oxidation, confirming a good sealing of the apertures allowing a flat and planar surface after chemical mechanical polishing.

positive photoresist (S1818, Shipley) by means of conventional photolithography. The SiN layers were opened [see Fig. 4(f)] using ICP anisotropic etching (Alcatel 601E: $\mathrm{C}_{2} \mathrm{~F}_{6} 20 \mathrm{sccm}$, $20{ }^{\circ} \mathrm{C}, 1800 \mathrm{~W}$ ). The front side patterns in the $\mathrm{SiN}$ form the membrane apertures and the backside patterned SiN forms the etch mask for the subsequent membrane releasing in potassium hydroxide (KOH) and BHF [see Fig. 4(g) and (h)].

A 3- $\mu$ m-thick low-temperature oxide (LTO) (LPCVD: $\mathrm{O}_{2}$ $220 \mathrm{sccm}, \mathrm{SiH}_{4} 137 \mathrm{sccm}, 425^{\circ} \mathrm{C}$, deposition rate $\left.120 \AA / \mathrm{min}\right)$ was deposited to protect the front-side during $\mathrm{KOH}$ etching [see Fig. 4(f)]. Then, $\mathrm{KOH}\left(40 \% \mathrm{wt}, 60^{\circ} \mathrm{C}\right)$ was used to etch into the bulk Si until a $20-\mu \mathrm{m}$-thick Si layer remains. The sacrificial $\mathrm{SiO}_{2}$ in the trenches as defined in a previous fabrication step can now be clearly seen as schematically depicted in Fig. 4(g). Finally, the remaining $\mathrm{SiO}_{2}$ trenches and the sacrificial $\mathrm{Si}$ pieces are removed by BHF $\left(\mathrm{NH}_{4} \mathrm{~F}(40 \%): \mathrm{HF}(49 \%)\right.$, ratio $\left.7: 1\right)$ resulting in Si-supported SiN membranes [see Fig. 4(h)].

Fig. 5 shows SEM images of finished Si-supported stencil membrane. The SEM image in Fig. 6(a) shows the Si-support structures following the membrane apertures while respecting a distance of $10 \mu \mathrm{m}$ from the aperture edge; this ensures the line-of-sight deposition process. Fig. 6(b) shows two SEM images of global membrane supports allowing for stable, large-area high-density membranes for use as deposition stencils, shadow masks, and filtration membranes.

\section{Characterization of Si-Supported Stencils}

To characterize and verify the stabilization effects of Si-supported SiN stencils on membrane deformation and surface structure definition, we performed a stencil deposition process through the previously described Si-supported stencil and through an identical, nonsupported stencil. The stencil shown in Fig. 6(a) was used to test and demonstrate the increased membrane performance and improved pattern replication because of its mechanically most unfavorable membrane geometry. The nonsupported stencil was fabricated using a standard (a)

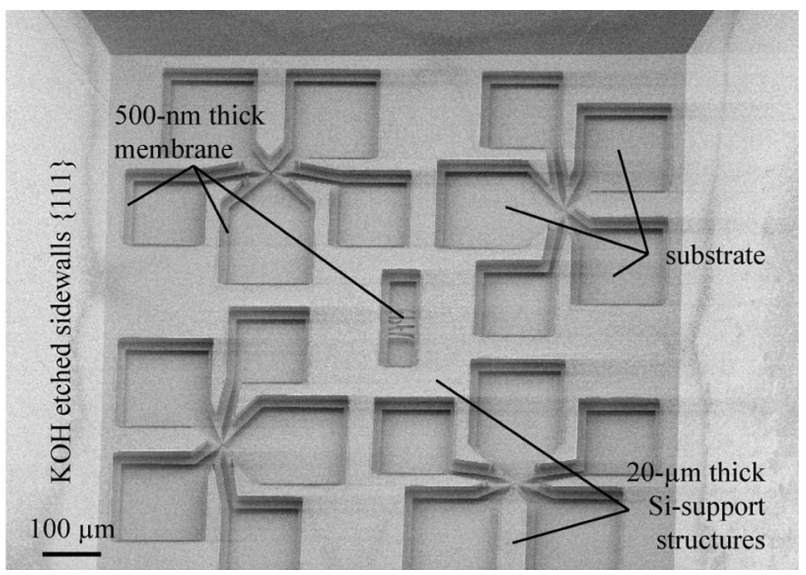

(b)

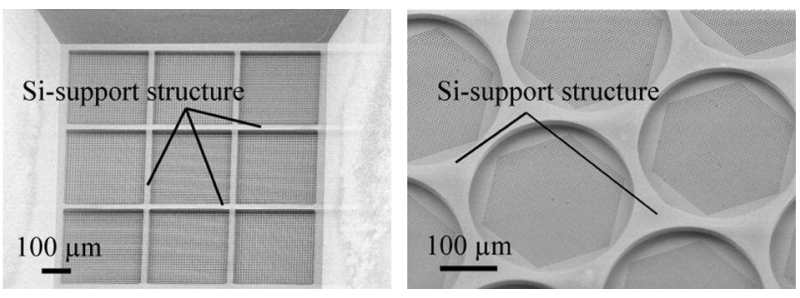

Fig. 6. SEMs of Si-supported SiN membranes on a substrate seen from the side facing the material flux. (a) The stabilization/Si-support structures follow the membrane apertures at a predefined distance allowing for a predetermined line-of-sight angle towards the evaporation source and minimizing membrane deformation. (b) SEM images of a global Si reinforced membranes.

stencil fabrication process [17]. These stencils contained membranes with exactly the same membrane thickness $(500 \mathrm{~nm})$ and apertures as the Si-supported membranes. A physical vapor deposition (PVD) evaporation system (Alcatel EVA 600) was used as deposition equipment for evaporation of a thin metal film. The process starts with positioning and mechanically clamping of both a Si-supported stencil and a nonsupported stencil to a substrate $(100 \mathrm{~mm}, 380-\mu \mathrm{m}$-thick $\mathrm{Si}$ wafer $)$. An additional reference wafer was used for the measurements of the thin-film residual stress by means of wafer curvature. Both the stencils/substrate combination and the reference wafer were centered and placed perpendicular at a distance of $50 \mathrm{~cm}$ from the material evaporation source. $\mathrm{Cr}$ was chosen as the test material because of its high internal stress as a thin film on SiN. A 35-nm-thick $\mathrm{Cr}$ film was evaporated using an electron gun (Alcatel EVA 600, $4 \cdot 10^{-7}$ mbar, $0.3 \mathrm{~kW}$, deposition rate: 3-4 $\AA$ / sec). The stress in the deposited $\mathrm{Cr}$ film was measured by wafer curvature (TENCOR FLX-2900) and was found to be $1900 \pm 100 \mathrm{MPa}$. Fig. 7 shows SEM images of both a nonsupported stencil membrane and a Si-supported stencil membrane after evaporation of 35-nm-thick Cr.

We analyzed systematically the stress-induced deflection by measuring in a SEM the bending of the free-standing membrane sections. Typically, the free-standing part of the stencil membrane (i.e., the thin silicon nitride part) extends $10 \mu \mathrm{m}$ from the Si-support structure. In some location on the stencil, however, the overhanging thin membrane part is longer due to a specific geometry at protruding membrane elements. Such a location is shown in Fig. 7(a) and (b) (Point A), where the nonsupported membrane overhangs the Si-support structure by about $47 \mu \mathrm{m}$. This location is particularly interesting to validate our 


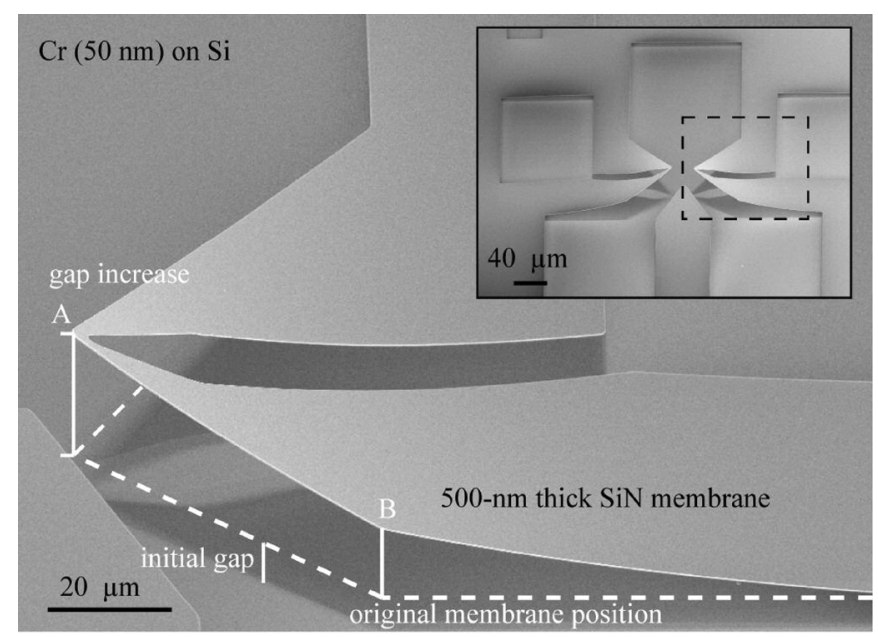

(a)

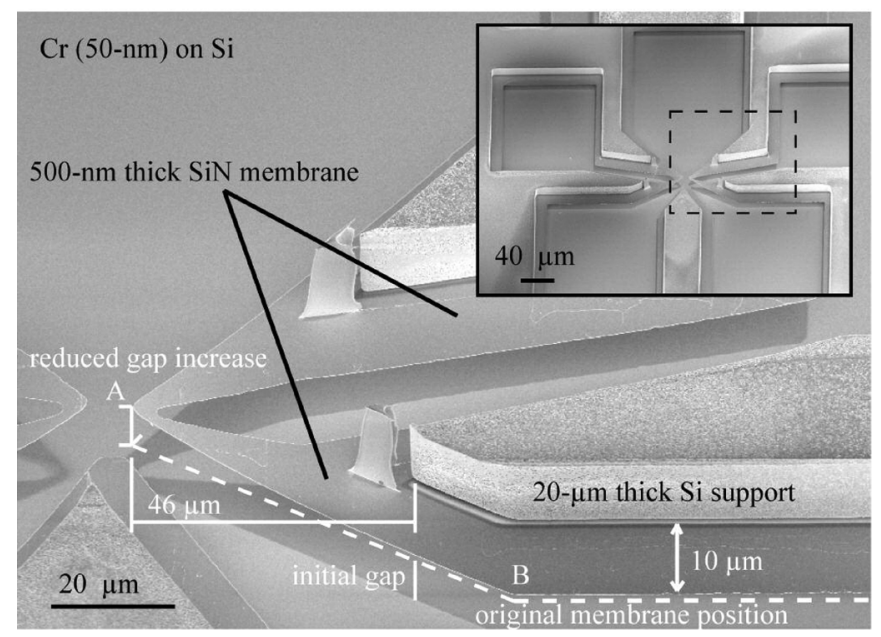

(b)

Fig. 7. Scanning electron micrographs of a nonsupported membrane and a Si-supported membrane after evaporation of $35 \mathrm{~nm}$ Cr. (a) Part of a nonsupported membrane, showing a relatively large ( $47 \mu \mathrm{m}$ at point A) out-of-plane deformation of the membrane. (b) Part of a Si-supported membrane, showing a significant reduction of the membrane deformation (68\% at point A and $89 \%$ at point B).

model and to demonstrate the stiffening effect of the Si-support structures to prevent stencil membrane bending. The following results were found: the measured maximum out-of-plane deformation of the non-supported membrane is $\sim 46.5 \mu \mathrm{m}$ [measured at Point $\mathrm{A}$ in respect to the initial membrane position in Fig. 7(a)] whereas it is only $\sim 15 \mu \mathrm{m}$ for the same geometry on the Si-supported membrane [Point A, Fig. 7(b)], which represents a $68 \%$ decrease in membrane deformation at this particular location (worst case). For the standard situation, where the $\mathrm{Si}$-support structures follow the silicon nitride membrane openings at a distance of $10 \mu \mathrm{m}$, a stencil deflection of only $1.5 \mu \mathrm{m}$ is measured [point B in Fig. 7(b)], yielding a deflection reduction of $>90 \%$ compared to the $\sim 26.5 \mu \mathrm{m}$ deflection of the unsupported membrane [Point B in Fig. 7(a)]. These values demonstrate clearly the positive effect of the $\mathrm{Si}$-support structures on the membrane bending.

In addition to the analysis of the stencils themselves upon deposition of physical vapor deposited material, we also studied

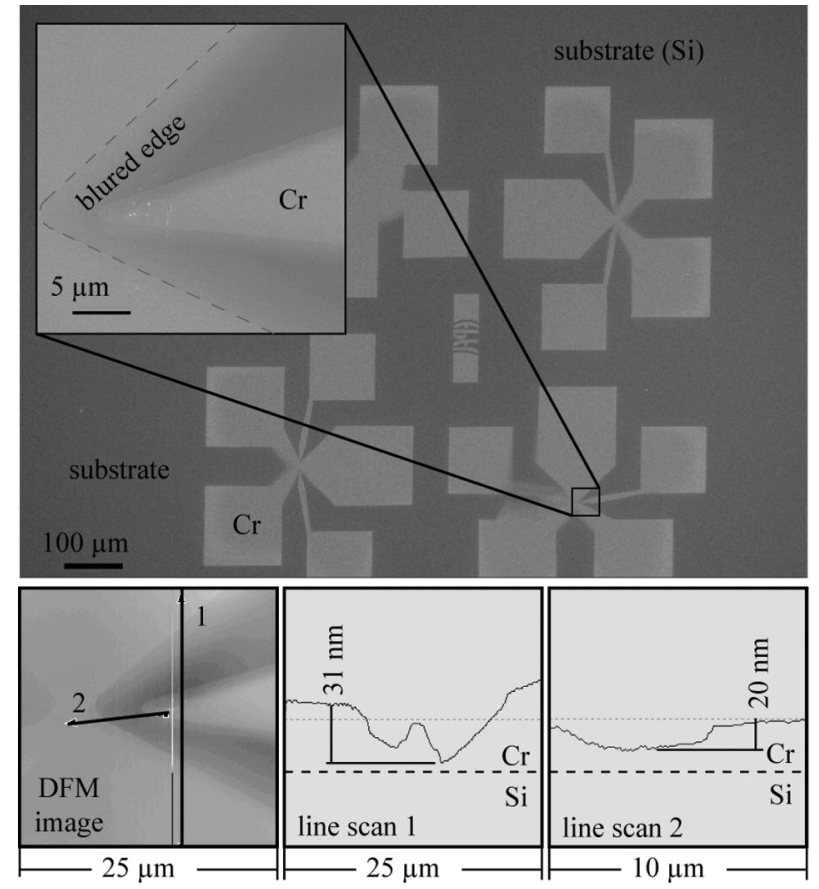

(a)
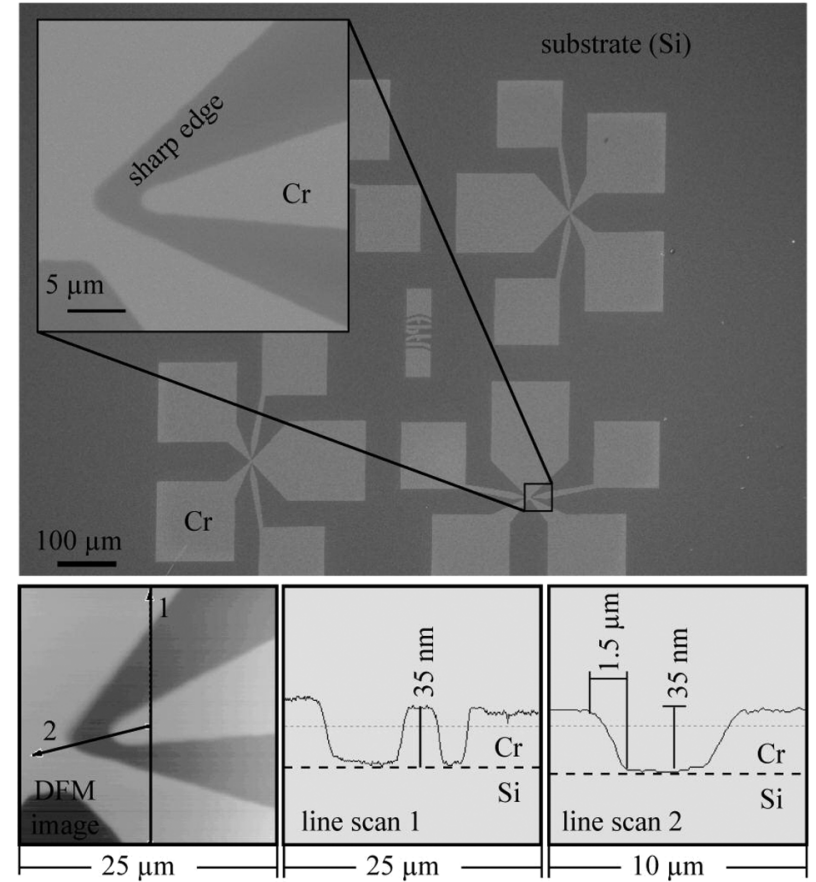

(b)

Fig. 8. SEM and DFM image of surface structures made by stencil deposition process. (a) For the nonstabilized stencil membranes, both SEM and DFM images show pattern blurring and an overlap of the deposited $\mathrm{Cr}$ resulting in a closed surface layer. (b) Both SEM and DFM images show excellent pattern definition due to the use of Si-supported membranes.

the effect of stencil bending on the surfaces structures. To this end, the stencils were removed from the substrate and the surface structures were analyzed by SEM and dynamic force microscopy (easyScan DFM, nanosurf). Fig. 8(a) and (b) shows SEM images and dynamic force microscope (DFM) images of 35-nm-thick Cr surface structures fabricated through both the 
nonsupported stencil membranes [see Fig. 8(a)] and the Si-supported stencil membranes [see Fig. 8(b)]. The stencils were separated from the substrate with an initial gap of approximately $15 \mu \mathrm{m}$.

The deposition of $\mathrm{Cr}$ through the nonsupported stencils yields patterns with blurred edges [see Fig. 8(a)] and an apparent film thickness of 20-nm [line scan 2 in Fig. 8(a)] in closely spaced surface patterns. We attribute this reduction of the measured step height to the overlap of the blurring (i.e., lateral spreading of deposited material through a stencil with large gap) coming from two adjacent, closely spaced patterns that are separated by only a few micrometers.

As expected through our earlier geometrical consideration, we find that the deposition of Cr film through the Si-supported stencils yields 35 -nm-thick individual structures with well-defined step edges [see Fig. 8(b)]. By inspecting the stencil geometry before and after deposition, we found an initial gap of $15 \mu \mathrm{m}$ and a final gap of approximately $30 \mu \mathrm{m}$. Fitting these values into our theoretical model that determines the step edge as a function of the gap yields a calculated step edge value of $300 \mathrm{~nm}$ [compared to the $1.5 \mu \mathrm{m}$ measured, shown in Fig. 8(b)]. We attribute this difference to the out-of-plane deformation of stencil membranes which not only affects the gap but also affects the lateral projection of the deposition flux, i.e., the membrane has a lateral deformation component. In our model, this value is on the order of $3 \mu \mathrm{m}$. However, we believe that the measured stencil deformation after deposition and unloading is bigger than the actual stencil deformation during deposition. We attribute this to temperature-related stress effects, which occur only once the evaporation is terminated and the stencil membrane with the deposited $\mathrm{Cr}$ layer has cooled down to room temperature. The resulting additional stress component due to the bimorph effect is the subject of further detailed investigations.

The results indicate that deposition through a nonsupported stencil may induce complete loss of pattern contrast, in particular for high-density micro- and nanopattern definition. In the case of the supported stencil, this effect is clearly reduced and full pattern contrast is obtained [line scan 2, Fig. 8(b)].

All the obtained results confirm that Si-supported stabilization structures significantly reduce out-of-plane deformation of the membrane and thus reduce the gap increase in stencil lithography. Both the SEM images and the DFM surface scans confirmed that Si-supported stencil membranes improve surface pattern definition in stencil lithography.

\section{CONCLUSION}

In this paper, the fabrication and application of Si-supported, mechanically reinforced, solid-state thin-film silicon-nitride membranes for improved stencil lithography have been demonstrated. The new microstencils incorporate in situ, local stabilization structures, increasing their moment of inertia up to a factor $10^{5}$. A variety of Si-supported membrane structures were fabricated that stabilize the stencil without conflicting with the conditions (i.e., line of sight criteria) for high-resolution stencil-based deposition process. We have systematically studied the behavior of non-supported and Si-supported membranes under influence of deposition induced stress and analyzed the resulting surface structures. The Si-supported membranes allow for a more accurate pattern transfer of the membrane apertures, compared to the standard unsupported stencil membranes. The proposed solution overcomes one of the major bottleneck that is currently limiting the use of thin mechanical solid-state membranes as high-resolution large-area shadow masks. The process used to stabilize the stencil membrane is based on standard MEMS technology allowing wafer-scale implementation and offers scaling-up potential. The stabilization of ultrathin membranes based on the proposed Si-rims is generic. Besides the application described in this paper for shadow deposition of physical vapor deposited thin films, it can be readily applied to other areas of interest, such as local ion implantation, microfluidic filtration, solid-state membranes for molecular sieves, etc. Stabilization of ultrathin membranes by local reinforcement structures as presented here reduces the risk of membrane damage during handling or operation, and in addition enables cleaning and recycling of used or contaminated membrane stencils, allowing for low-cost applications in the field of membrane-based stencil lithography.

\section{ACKNOWLEDGMENT}

The authors are pleased to acknowledge the EPFL Center of Micro-Nano-Technology (CMI) and its entire staff as well as their colleagues at the Microsystems Laboratory for their very valuable discussions and help.

\section{REFERENCES}

[1] S. Y. Chou, P. R. Krauss, and P. J. Renstrom, "Nanoimprint lithography," J. Vac. Sci. Technol. B, vol. 14, no. 6, pp. 4129-4133, 1996.

[2] S. Johnson et al., "Step and flash imprint lithography modeling and process development," J. Photopolymer Sci. Technol., vol. 17, pp. 417-419, 2004.

[3] B. Michel et al., "Printing meets lithography: soft approaches to high-resolution patterning," IBM J. Res. Develop., vol. 45, no. 5, pp. 697-719, 2001

[4] A. Kumar and G. M. Whitesides, "Features of gold having micrometer to centimeter dimensions can be formed through a combination of stamping with an elastomeric stamp and an alkanethiol "ink" followed by chemical etching," Appl. Phys. Lett., vol. 63, no. 14, pp. 2002-2004, 1993.

[5] R. D. Piner et al., ““Dip-pen” nanolithography,” Science, vol. 283, no. 5402, pp. 661-663, 1999.

[6] A. Meister et al., "Nanoscale dispensing of liquids through cantilevered probes," Microelectron. Eng., vol. 67-68, pp. 644-650, 2003.

[7] G. M. Kim, M. A. F. van den Boogaart, and J. Brugger, "Fabrication and application of a full wafer size micro/nanostencil for multiple length-scale surface patterning," Microelectron. Eng., vol. 67-68, pp. 609-614, 2003

[8] J. Köhler et al., "Direct growth of nanostructures by deposition through an $\mathrm{Si}_{3} \mathrm{~N}_{4}$ shadow mask," Phys. E, Low-Dimensional Syst. Nanostruct., vol. 4, no. 3, pp. 196-200, 1999.

[9] M. M. Deshmukh et al., "Nanofabrication using a stencil mask," Appl. Phys. Lett., vol. 75, no. 11, pp. 1631-1633, 1999.

[10] J. Brugger et al., "Resistless patterning of sub-micron structures by evaporation through nanostencils," Microelectron. Eng., vol. 53, no. $1-4$, pp. 403-405, 2000.

[11] M. A. F. van den Boogaart et al., "Corrugated membranes for improved pattern definition with micro/nanostencil lithography," Sens. Actuators A, Phys., to be published.

[12] E. A. Speets et al., "Fabrication of arrays of gold islands on self-assembled monolayers using pulsed laser deposition through nanosieves," Nano Lett., vol. 4, no. 5, pp. 841-844, 2004.

[13] M. Graff et al., "Microstenciling: A generic technology for microscale patterning of vapor deposited materials," J. Microelectromech. Syst., vol. 13, no. 6, pp. 956-962, 2004.

[14] T. Shibata et al., "Stencil mask ion implantation technology," IEEE Trans. Semicond. Manufact., vol. 15, no. 2, pp. 183-188, 2002. 
[15] A. Tixier et al., "A silicon shadow mask for deposition on isolated areas," J. Micromech. Microeng., vol. 10, no. 2, pp. 157-162, 2000.

[16] J. Arcamone et al., "Pattern recovery by dry etching of gap-induced blurring in nanostencil lithography," in Int. Conf. Micro- Nano Eng. (MNE 2005), Vienna, Austria, 2005.

[17] M. A. F. van den Boogaart et al., "DUV-MEMS stencils for highthroughput resistless patterning of mesoscopic structures," J. Vac. Sci. Technol. B, vol. 22, no. 6, pp. 3174-3177, 2004.

[18] M. Kölbel et al., "Self-assembled monolayer coatings on nanostencils for the reduction of materials adhesion," Adv. Functional Mater., vol. 13, no. 3, pp. 219-224, 2003.

[19] M. Ohring, The Material Science of Thin Films. New York: Academic, 1992.

[20] S. Yotsuya and T. Makigaki, "The patterning of electric circuits by mask-deposition technology," in Proc. MEMS 2006, Istanbul, Turkey, 2006.

[21] G. J. Burger et al., "High-resolution shadow-mask patterning in deep holes and its application to an electrical wafer feed-through," Sens. Actuators A, Phys., vol. 54, no. 1-3, pp. 669-673, 1996.

[22] S. Kuiper et al., "Wet and dry etching techniques for the release of sub-micrometre perforated membranes," J. Micromech. Microeng., vol. 10, pp. 171-174, 2000.

[23] S. Kuiper et al., "Development and applications of very high flux microfiltration membranes," J. Mem. Sci., vol. 150, pp. 1-8, 1998.

[24] R. Hammer, "Solving the "stencil" problem in vacuum deposition masks using rib-supported structures," J. Vac. Sci. Technol., vol. 14, no. 5 , pp. $1208-1210,1977$.

[25] G. G. Stoney, "The tension of metallic films deposited by electrolysis," Proc. Roy. Soc. London, vol. A82, no. 553, p. 172, 1909.

[26] W. C. Young and R. G. Budynas, Roark's Formulas for Stress and Strain. New York: McGraw-Hill, 2002.

Marc A. F. van den Boogaart received the B.Sc. degree in precision engineering with a specialization in microengineering from the Hogeschool van Utrecht, Utrecht, The Netherlands, in 2000.

From 2000 to 2002, he worked on the development of thin-film microelectrode arrays, designed for interaction with cultured neurons at the University of Twente, Enschede, The Netherlands. He received the Ph.D. degree from Ecole
Polytechnique Fédérale de Lausanne (EPFL), Lausanne, Switzerland, in 2006 for his work on the development of stencil lithography, a large-scale and resist-less micro and nano patterning technique. He is currently an active as Researcher at Ecole Polytechnique Fédérale de Lausanne (EPFL) and within the EU Sixth Framework IP NaPa.

Lianne M. Doeswijk received the M.Sc. degree in applied physics with a specialization in heat transfer and fluid dynamics from the University of Twente, Enschede, The Netherlands, in 1997. She received the Ph.D. degree from the University of Twente in 2002 for her dissertation: "Pulsed Laser Deposition of Oxide Films on Silicon: Exploring their Passivating Qualities."

From 2004 to 2006, she worked on the development of stencil lithography at the Ecole Polytechnique Fédérale de Lausanne (EPFL), Lausanne, Switzerland. Currently, she is working as the Program Coordinator for the Dutch innovationoriented program (IOP) Photonic Devices.

Jürgen Brugger received the Ph.D. degree from the University of Neuchatel, Switzerland, in 1995 for a work on microfabricated tools for the atomic force microscope, which included a one-year period at the Hitachi Central Research Laboratories, Tokyo, Japan.

He then joined the IBM Zurich Research Laboratory in Rüschlikon, Switzerland. From 1998 to 2001, he was directing the "NanoLink" Strategic Research Orientations at the MESA+ Research Institute, University of Twente, The Netherlands. In September 2001, he was appointed Assistant Professor "tenure track" at the EPFL. He is co-inventor of eight patents. His main professional interests include the development of new tools for nanoscience and engineering techniques at the mesoscopic length-scale; in particular, to develop methods for accessing the nanometer scale (top-down), and to combine them with self-assembly strategies (bottom-up), and to bridge life-science with solid-state devices at the submicrometer scale.

Dr. Brugger is the elected management board member of the EU Network of Excellence for Nanoelectronics Phantoms, Steering Board Member of the EU Sixth Framework IP NaPa, member of the EU NoE Nano-2-Life, and the Associated Editor of the Journal of Nanoscience and Nanotechnology. He received two IBM research awards. 